\title{
Autoeficacia, participación social y percepción de los servicios universitarios según el sexo
}

\author{
Self-Efficacy, Social Participation, and Perception Regarding \\ University Services by Sex
}

\author{
María Elena Rivera Heredia ${ }^{a}$, Miriam Martínez Fuentes ${ }^{a}$, Fabiola González Betanzos ${ }^{a}$, \& Miriam Anahí Salazar García ${ }^{\mathrm{b}}$ \\ ${ }^{a}$ Universidad Michoacana de San Nicolás de Hidalgo, Michoacán, México \\ ${ }^{b}$ Instituto Mexicano de Terapias Breves, Ciudad de México, México
}

\begin{abstract}
Resumen: El objetivo de esta investigación fue analizar la autoeficacia, la participación social y la percepción de los servicios universitarios entre los hombres y las mujeres que estudian educación superior en una universidad de Michoacán, México. El estudio fue cuantitativo y transversal. Participaron 587 estudiantes universitarios, quienes respondieron a una Escala de Autoeficacia, a otra de Participación Social y a un Cuestionario sobre el Uso y Satisfacción de los Servicios de Atención Estudiantil. Se encontraron diferencias significativas entre hombres y mujeres en la autoeficacia deportiva y en la autoeficacia para resistir la presión. También se identificó mayor participación social en los hombres que en las mujeres y escaso contacto con la oferta de servicios universitarios en ambos. En conclusión, se requiere mayor difusión de los servicios universitarios de atención estudiantil, además de promover que estos aumenten la autoeficacia y la participación social, tanto en hombres como en mujeres.
\end{abstract}

Palabras clave: estudiantes universitarios, autoeficacia, participación social, servicios, educación superior.
Abstract: The aim of this work was to analyze the self-efficacy, social participation and perception of the university services between men and women who study at a higher education institution in Michoacán, Mexico. The study was quantitative and transversal. A total of 587 university students participated. They answered a Self-Efficacy Scale, a Social Participation Scale, and a Questionnaire of the Use and Satisfaction of the Services Delivered to the Students. There were found differences in pressure resistance and sport self-efficacy. More social participation was found in men than in women, and a low contact with the university services in both groups. In conclusion, more diffusion of the university services is required to call the attention of the students, and to promote these services in order to increase the perception of self-efficacy, and social participation in men and in women.

Keywords: university students, self-efficacy, social participation, services, higher education.

Se agradece el apoyo brindado por la Coordinación de Investigación Científica de la Universidad Michoacana de San Nicolás de Hidalgo.

Contacto: M. E. Rivera Heredia. Universidad Michoacana de San Nicolás de Hidalgo. Bosque de Cerezos 143, Bosques Tres Marías, C.P. 58200 Morelia, Michoacán, México. Correo electrónico: dip.umsnh.maelenarivera@ gmail.com

Cómo citar: Rivera Heredia, M. E., Martínez Fuentes, M., González Betanzos, F., \& Salazar García, M. A. (2016). Autoeficacia, participación social y percepción de los servicios universitarios según el sexo. Revista de Psicología, 25(2), 1-16. http://dx.doi.org/10.5354/0719-0581.2017.44842 


\section{Introducción}

En diferentes partes del mundo una de las principales problemáticas que presentan los estudiantes universitarios durante la realización de sus estudios de nivel superior es la reprobación y la deserción (González-Betanzos, Rivera-Heredia, \& García-Rangel, 2016). La evidencia científica ha mostrado que la continuidad de los estudios universitarios y el alto desempeño académico aunado a las variables de capacidad intelectual, también se relacionan con las creencias de los estudiantes sobre sí mismos y las expectativas favorables y de autoconfianza que puedan tener sobre su desempeño a futuro (Feldman \& Kubota, 2015; Richardson, Abraham, \& Bond, 2012).

Debido a esto, el aporte de la psicología para clarificar con mayor precisión las diferencias y similitudes que existen en la autoeficacia entre los hombres y las mujeres de diferentes contextos económicos y socioculturales (Dever \& Kim, 2016; Hen \& Goroshit, 2014; MacPhee, Farro, \& Canetto, 2013), permitirá contar con información que pueda utilizarse para disminuir los problemas de reprobación y deserción escolar, así como para generar niveles más altos de bienestar en los estudiantes. Además, se requiere información sobre cómo perciben los jóvenes los servicios que les proporcionan las universidades, el uso que le dan a los mismos y sus niveles de satisfacción, puesto que estos elementos también proporcionan información relevante que puede estar vinculada a su desempeño y trayectoria académica en la institución educativa.

Como es sabido, durante su permanencia en la universidad, los estudiantes están expuestos a exigencias académicas y de la vida cotidiana, las que pueden poner en riesgo su buen desempeño, especialmente cuando desconfían de sus capacidades y potencialidades (Morony, Kleitman, Ping Lee, \& Stankov, 2013; Stankov, Lee, Luo, \& Hogan, 2012). Por esta razón, el fortalecimiento de los procesos cognitivos-motivacionales funciona como estrategia para generar factores protectores ante los desafíos académicos, la deserción escolar y el riesgo al fracaso académico (Dapelo Pellarano \& Matus Jara, 2013). Uno de dichos procesos es la autoeficacia, definida por Bandura (1977) como "las creencias en la propia capacidad para organizar y ejecutar las acciones requeridas para manejar las situaciones futuras" (p. 2). Esta es un recurso psicológico de tipo cognitivo, que se relaciona con la percepción concebida para lograr realizar una actividad o enfrentar situaciones o problemas (Rivera Heredia \& Pérez Padilla, 2012).

La percepción de autoeficacia es elemental en la competencia humana, ya que se relaciona con patrones de pensamientos que pueden aumentar la motivación, el esfuerzo y la persistencia, así como disminuir alteraciones emocionales (Flores León, González-Celis Rangel, \& Valencia Ortiz, 2010; Qualter et al., 2015). En consecuencia, los estudiantes con autoeficacia alta se ponen objetivos y retos que implican desafíos, los cuales utilizan como una forma de obtener nuevos conocimientos y perfeccionamiento (Komarraju \& Nadler, 2013).

Generalmente, la autoeficacia se estudia en diferentes áreas del funcionamiento humano y se ha demostrado su relevancia e influencia en diversos campos, de ahí que esta se evalúa de forma específica, por ejemplo: autoeficacia académica, autoeficacia deportiva o autoeficacia social (Blanco, Aguirre, Barrón, \& Blanco, 2016; Peinado, Ornelas, Blanco, \& González, 2015; Reigal Garrido, Videra Gar- 
cía, Martín Tamayo, \& Juárez Ruiz de Mier, 2013); aunque también en los últimos años se han hecho propuestas para medir la autoeficacia de manera general (Aguirre, Blanco, Rodríguez-Villalobos, \& Ornelas, 2015; Brenlla, Aranguren, Rossaro, \& Vázquez, 2010; Espada, González, Orgilés, Carballo, \& Piqueras, 2012).

Desde el área de la psicología educativa, el estudio de la autoeficacia y su asociación con el desempeño escolar pueden contribuir a mejorar las prácticas pedagógicas y de enseñanza (Chávez, Ornelas, De Rueda, \& Jasso, 2012; Dever \& Kim, 2016). Además, la percepción de autoeficacia se puede mejorar en las instituciones si se orientan los servicios universitarios hacia la solución de problemas, donde los estudiantes pueden encontrar un apoyo para sortear las diferentes situaciones que se les presentan. Asimismo, a partir de la percepción de autoeficacia se busca ayudar a los estudiantes a encontrar su vocación tanto profesional como laboral (Pérez \& Cupani, 2008). Por tanto, el objetivo de este trabajo fue analizar la autoeficacia, la participación social y la percepción de los servicios universitarios, considerando las diferencias y similitudes entre los hombres y las mujeres que estudian educación superior en una universidad de Michoacán, México.

Entre los diferentes tipos de autoeficacia, la académica es la más relacionada con el desenvolvimiento y desempeño académico de los estudiantes (Feldman \& Kubota, 2015; Richardson et al., 2012). De igual manera, el estudio de la autoeficacia académica permite entender por qué los estudiantes con las mismas habilidades y conocimientos presentan conductas y/o resultados diferentes a los esperados (Alegre, 2013). Este recurso psicológico en el ámbito académico favorece el proceso en el que los estudiantes buscan y encuentran estrategias para estudiar, aprender y realizar tareas escolares. En este caso, la autoeficacia juega un papel de factor protector, porque los estudiantes se convierten en individuos más competentes y reducen las posibilidades de abandono escolar (Weng, Cheong, \& Cheong, 2010). En consecuencia, los estudiantes con creencias de autoeficacia altas conciben las tareas académicas como retos y tienden a sentirse más motivados para alcanzar sus metas; en cambio, los estudiantes con baja percepción de autoeficacia conciben el entorno académico como una amenaza, tienden a dudar de sus capacidades y creen que las cosas son más difíciles de lo que realmente son (Blanco Vega, Ornelas Contreras, Aguirre Chávez, \& Guedea Delgado, 2012; Cabanach, Valle, Rodríguez, Piñeiro, \& González, 2010). Además, los estudiantes con una baja percepción de autoeficacia académica son más propensos a presentar estrés, burnout académico (Caballero Domínguez, Hederich, \& Palacio Sañudo, 2010) o ansiedad (Rouxel, 1999).

Por su parte, los factores sociales impactan en el grado de autoeficacia académica. Uno de estos es de tipo cultural, que se relaciona con las creencias que dictan cuáles son las actividades propias de los hombres y de las mujeres. Estas creencias repercuten en el grado de estudios y la elección de carrera (Abdul Gafoor \& Muhammed Ashraf, 2012). Las investigaciones que presentan estudios comparativos entre hombres $y$ mujeres demuestran que existen diferencias de autoeficacia académica entre ellos; por ejemplo, las mujeres, por lo general, presentan niveles más altos de autoeficacia académica y mayores deseos de terminar sus estudios que los hombres (Saunders, 
Davis, Williams, \& Williams, 2004). Sin embargo, son pocas las mujeres que desempeñan ocupaciones típicamente masculinas, porque se han perpetuado creencias de baja eficacia de la mujer para desenvolverse en carreras tradicionalmente masculinas o que socialmente son vistas como áreas propias de los varones (Hackett \& Betz, 1981).

Dado que la salud se crea y se vive día a día en los centros de enseñanza, de trabajo y de recreo, las instituciones educativas, como segundo hogar de los estudiantes, deben colaborar con el bienestar de los jóvenes (Calvo Muñoz \& Gómez Conesa, 2013). La gran mayoría de las enfermedades que podrían presentar los universitarios están relacionadas con niveles altos de estrés y ansiedad ante el aumento de demandas académicas ( $\mathrm{Pu}$ lido Rull et al., 2011). En este sentido, la práctica de actividades físicas y recreativas ayuda a disminuir los niveles de estrés y favorece la calidad de vida de los estudiantes universitarios (Hernández Zamora \& Romero Pedraza, 2010). Sin embargo, el nivel de actividad física de los universitarios tiende a ser bajo o nulo. Esta situación es aún más preocupante en el caso de las mujeres que tienden a ser más sedentarias (Varela, Duarte, Salazar, Lema, \& Tamayo, 2011). Así, la percepción de autoeficacia deportiva varía entre hombres y mujeres: generalmente, los hombres tienen niveles más altos de autoeficacia deportiva que las mujeres (Abalde Amoedo \& Pino Juste, 2016). Esta diferencia podría relacionarse con creencias de que el deporte se relaciona con la masculinidad y no con la femineidad ( $\mathrm{Pa}-$ vón Lores \& Moreno Murcia, 2008).

El apoyo social es un factor protector de la salud mental en los estudiantes, quienes se enfrentan a cambios constantes. Este conlleva un mayor bienestar psicológico grupal y, a su vez, ese bienestar permite que los jóvenes desarrollen estrategias de afrontamiento o solución de problemas eficaces (González Barrón, Montoya Castilla, Casullo, \& Bernabéu Verdú, 2002). Estas estrategias son habilidades sociales que propician las conductas de intercambio, por ejemplo: comunicación, empatía, asertividad, control de la ira y prosocialidad (Rosa-Rodríguez, Negrón Cartagena, Maldonado Peña, Quiñones Berrios, \& Toledo Osorio, 2015). De este modo, la autoeficacia social como habilidad cognitiva permite a los estudiantes tener mayor asertividad y confianza para interrelacionarse, solucionar problemas con pares, y evitar los problemas de comunicación, así como la evasión de situaciones (Morales Rodríguez, Benítez Hernández, \& Agustín Santos, 2013).

Generalmente, la población joven no tiende a presentar enfermedades o trastornos que amenacen su vida, pero la adopción de malos hábitos y el estilo de vida de algunos jóvenes representan un riesgo para su salud en etapas posteriores, por ejemplo: el consumo de tabaco, sustancias adictivas y alcohol (Rhodes \& Jason, 1990). El riesgo de morbilidad y mortalidad actual es mayor en aquellos que tienen conductas de riesgo (Pérez Padilla, Ponce Rojo, Hernández Contreras, \& Márquez Muñoz, 2010). Existen factores protectores de tipo social y psicológico que influyen en la toma de decisión y en el autocontrol para consumir o no una sustancia (Varela Arévalo, Salazar Torres, Cáceres de Rodríguez, \& Tovar Cuevas, 2007). La percepción de autoeficacia para resistir la presión puede ayudar a disminuir o dejar de consumir sustancias (Chicharro Romero, Pedrero Pérez, \& Pérez López, 2007). Además, esta aumenta la confianza, disminuye los conflictos sociales y evita el consumo que inicia por simple aceptación social. 
Algunos programas de intervención que trabajan con el fortalecimiento de la autoeficacia han mostrado un impacto positivo (Lira-Mandujano, González-Betanzos, Carrascoza Venegas, Ayala, \& CruzMorales, 2009).

La participación social implica el involucramiento del individuo en actividades de convivencia social, ya sea de tipo deportivo, artístico o cultural, de acción en grupos organizados, entre otros. Esta puede llevarse a cabo de manera presencial o virtual, y puede aplicarse a diferentes contextos y escenarios. Por ejemplo, podemos ubicarla en el campo de la educación (Estrada Ruiz, 2010; Perales Mejía \& Escobedo Carrillo, 2016), en el de la defensa de los derechos humanos y ciudadanía (López González \& Anaya Rodríguez, 2016) o en el del deporte (Pérez Moreno \& Álvarez Hernández, 2015).

Finalmente, para que un estudiante cumpla sus objetivos no solo necesita autoeficacia, sino que también debe poseer las habilidades, incentivos y recursos materiales necesarios (Bandura, 1986). El apoyo a los estudiantes es indispensable para que generen una sensación de dominio, aprendan de ellos mismos, adquieran experiencias positivas y desarrollen sus habilidades hasta culminar en el logro personal. Las universidades y los docentes deben asumir la responsabilidad de motivar y de preparar a los estudiantes para hacerlos sentir seguros de sí mismos, capaces de perseguir y conseguir sus propios objetivos (Blanco Vega et al., 2012). Por ello, esta investigación pretende generar conocimiento para retroalimentar a las instituciones educativas sobre el estado de la autoeficacia de los estudiantes y su participación en actividades sociales e institucionales.

\section{Método}

\section{Participantes}

Un total de 587 estudiantes participaron en este estudio, de los cuales $357(60,8 \%)$ eran mujeres y $230(39,2 \%)$, hombres, con un rango de edad entre 16 y 32 años $(M=20,1$ años; $D E=1,96)$. Los estudiantes pertenecen a diversas licenciaturas de tres poblaciones distintas en las que se encuentra la Universidad Michoacana de San Nicolás de Hidalgo: Morelia, Ciudad Hidalgo y Lázaro Cárdenas, Michoacán. Se encontraban cursando primer semestre 208 (35,4\%), tercer semestre 155 $(26,4 \%)$, quinto semestre $53(9 \%)$, séptimo semestre $57(9,7 \%)$ y noveno semestre $63(10,7 \%)$ estudiantes, un total de 51 estudiantes no informó sobre el semestre que estaba cursando.

\section{Instrumentos}

Escala de Autoeficacia. Se aplicaron 16 reactivos de la adaptación al castellano de la Escala de Autoeficacia realizada por Carrasco Ortiz \& Del Barrio Gándara (2002). Esta evalúa la percepción de eficacia que el estudiante posee en los distintos ámbitos. Se trata de una escala tipo Likert con cuatro opciones de respuesta: muy bueno, bueno, malo, muy malo. Para la presente investigación se extrajeron cuatro factores que miden: autoeficacia académica $(\alpha=, 82)$, deportiva $(\alpha=, 85)$, para afrontar problemas $(\alpha=, 8)$, y para resistir la presión social $(\alpha=, 8)$. La confiabilidad total de la escala fue de ,83.

Escala de Participación Social. Propuesta por Rivera-Heredia (Rivera Heredia, Obregón Velasco, \& Ayala Mira, 2012), consta de nueve reactivos y tiene una estructura tipo Likert con cuatro opciones de respuesta: casi siempre, algunas 
veces, rara vez y casi nunca. Su confiabilidad es de ,88.

Cuestionario sobre el Uso y Satisfacción de los Servicios de Atención Estudiantil. Este cuestionario se diseñó especialmente para la presente investigación. Consta de preguntas cerradas que permiten identificar si se ha tenido o no acceso a una serie de servicios universitarios y se califica el nivel de satisfacción que tuvieron con los mismos, en una escala del 0 al 10, siendo el cero el nivel mínimo de satisfacción y el 10 el máximo.

\section{Procedimiento}

Las escalas fueron aplicadas dentro de las instalaciones educativas donde los jóvenes participantes realizan sus estudios. Se les explicó el objetivo de la investigación y la confidencialidad con la que se guardaría la información recabada. Todos ellos firmaron una carta de consentimiento informado.

\section{Análisis de los resultados}

Se realizaron análisis descriptivos (de medias y frecuencias) e inferenciales (prueba $t$ para comparación de dos muestras independientes). La información recabada se capturó en el paquete estadístico para las ciencias sociales SPSS versión 17.

\section{Resultados}

En la tabla 1 se presentan los resultados para los hombres y las mujeres en cada una de las dimensiones de autoeficacia, así como los resultados de las diferencias entre ellos. En términos generales se observa que la percepción de autoeficacia de los estudiantes varía entre moderada y alta en cada una de las dimensiones. Entre los resultados generales se obtuvieron diferencias significativas por sexo en las dimensiones autoeficacia deportiva y autoeficacia para resistir la presión social; respectivamente, los hombres se perciben como mejores en el ámbito deportivo y las mujeres se perciben mejores para resistir la presión social.

Respecto a los resultados obtenidos en la dimensión de autoeficacia deportiva, las medias indican que los estudiantes consideran estar en un nivel medio en el ámbito deportivo. Las mujeres tienen una menor autoeficacia deportiva $(M=2,58$, $D E=0,74)$ que los hombres $(M=2,93$, $D E=0,76)$. En este caso, la diferencia sí es significativa $(t[548]=5,350, p<, 05)$, aunque el tamaño del efecto es pequeño ( $d$ Cohen $=0,22)$. En el análisis por ítems, ellos se consideran mejor para ejercitarse y para formar parte de equipos deportivos (figura 1).

Tabla 1

Comparación de las dimensiones de autoeficacia entre hombres y mujeres

\begin{tabular}{|c|c|c|c|c|c|c|c|c|}
\hline \multirow{2}{*}{ Autoeficacia } & \multicolumn{2}{|c|}{ Hombre } & \multicolumn{2}{|c|}{ Mujer } & \multirow{2}{*}{$g l$} & \multirow{2}{*}{$t$} & \multirow{2}{*}{$p$} & \multirow{2}{*}{$d$ Cohen } \\
\hline & $M$ & $D E$ & $M$ & $D E$ & & & & \\
\hline Académica & 2,88 & 0,49 & 2,93 & 0,46 & 538 & $-1,145$ & ,25 &, 05 \\
\hline Deportiva & 2,93 & 0,76 & 2,58 & 0,74 & 548 & 5,350 & $<, 05$ &, 22 \\
\hline Enfrentar problemas & 3,17 & 0,64 & 3,20 & 0,61 & 556 &,- 551 & ,59 &, 02 \\
\hline Resistir la presión social & 3,10 & 0,73 & 3,29 & 0,71 & 545 & $-2,963$ & $<, 05$ &, 13 \\
\hline
\end{tabular}

Nota: $M=$ media $; D E=$ desviación estándar $; g l=$ grados de libertad; $t=\mathrm{t}$ de student; $p=$ probabilidad; $d$ Cohen $=$ tamaño del efecto . 


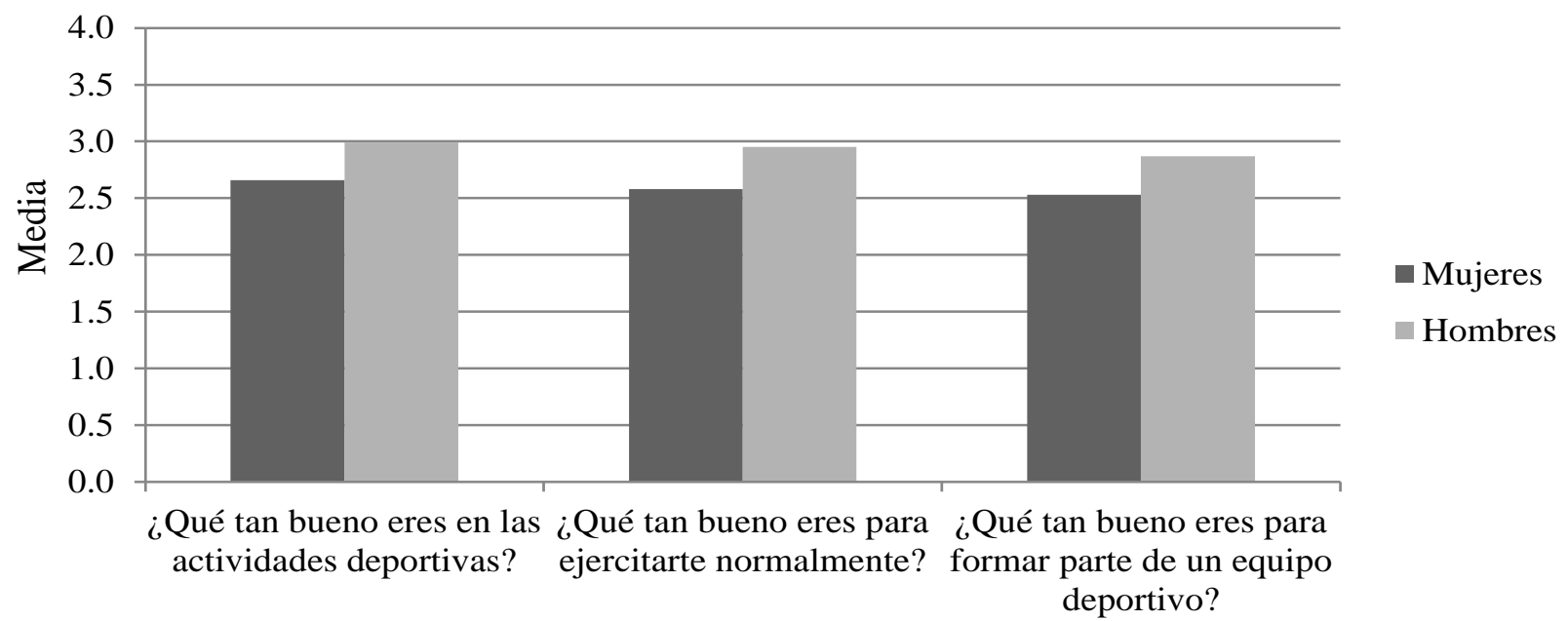

Figura 1. Autoeficacia deportiva entre hombres y mujeres.

Con relación a los resultados obtenidos en la dimensión de autoeficacia para resistir la presión social, las medias indican que las mujeres $(M=3,29, D E=0,71)$ tienen puntajes significativamente más altos que los hombres $(M=3,10, D E=0,73)$. En este caso, la diferencia sí es significativa $(t[545]=-2,963, p<, 05)$, aunque el tamaño del efecto es pequeño $(d$ Cohen $=$ 0,13). En el análisis por ítems no se en- contraron diferencias significativas entre hombres y mujeres (ver figura 2).

El análisis sobre la participación de los estudiantes en actividades deportivas y artísticas señala frecuencias bajas: solo el $30 \%$ suele participar en actividades deportivas y la respuesta de participación en actividades artísticas fue de un $21 \%$.

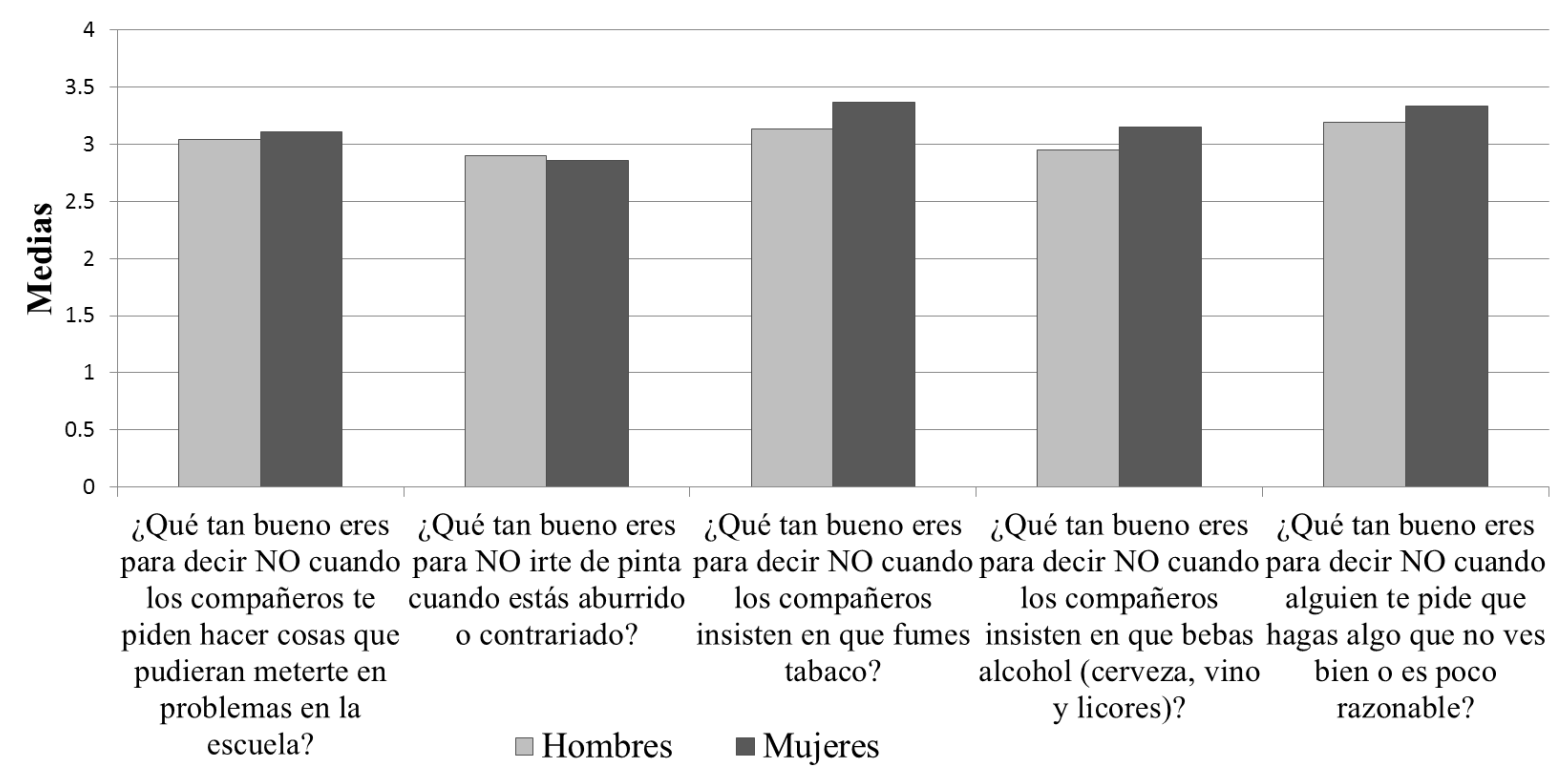

Figura 2. Autoeficacia para resistir la presión social entre hombres y mujeres. 
Se encontraron niveles bajos de participación social en ambos grupos de estudiantes universitarios. En esta dimensión, los hombres reportaron una mayor participación en eventos públicos cantando, bailando, declamando, actuando o en alguna otra actividad artística; de igual forma, participaron en mayor medida que las mujeres en actividades deportivas y en actividades culturales (figura 3 ).

Por otra parte, la frecuencia, los porcentajes de uso y la evaluación de satisfac- ción de cada uno de los programas de apoyo de los servicios universitarios se presentan en la tabla 2. Los porcentajes de uso del servicio se encuentran entre un $1,02 \%$, que es el apoyo a estudiantes indígenas, y un 16,9\%, que son las actividades artísticas. Esto indica que la frecuencia con la que utilizan estos servicios es muy baja. Sin embargo, los estudiantes que sí utilizaron los servicios los califican con altos niveles de satisfacción.

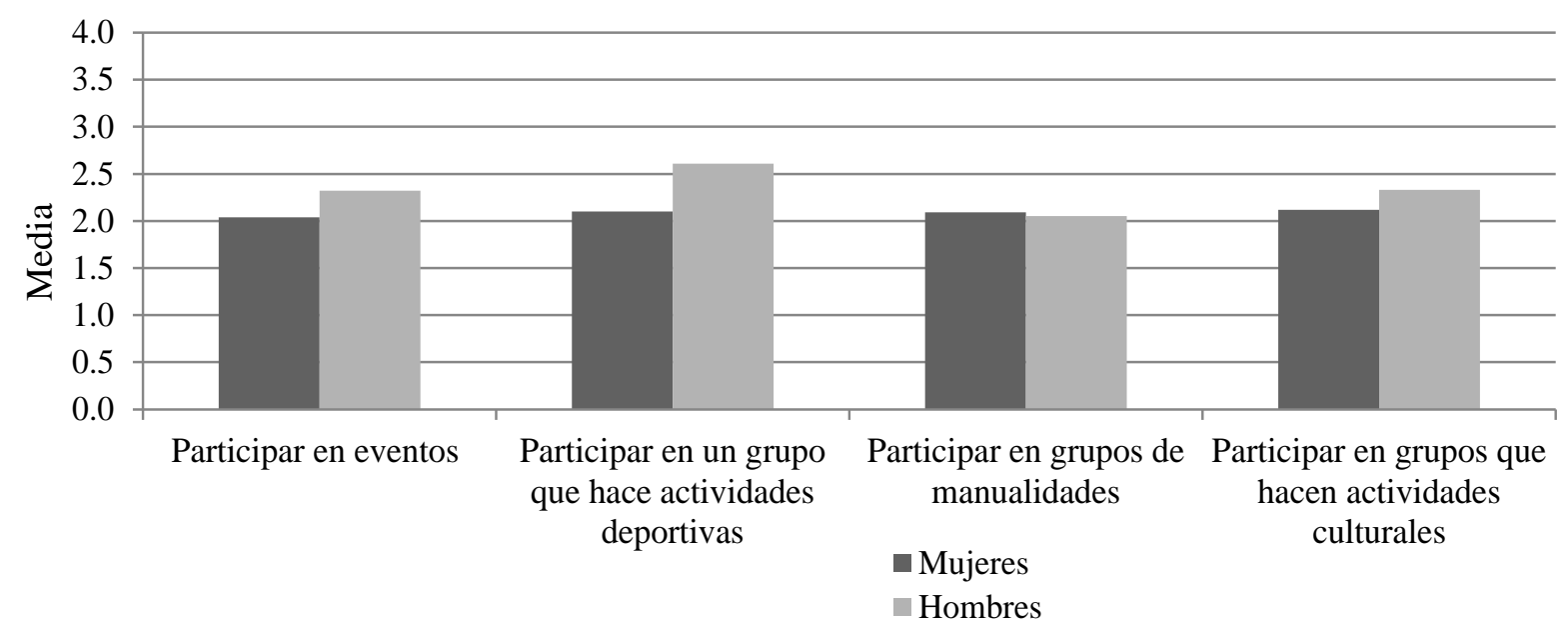

Figura 3. Promedio de participación social en diferentes actividades según el sexo.

Tabla 2

Evaluación de los servicios universitarios

\begin{tabular}{lcccc}
\hline \multicolumn{1}{c}{ Programa o servicio } & $\begin{array}{c}\text { Estudiantes que han } \\
\text { recibido el servicio }\end{array}$ & $\%$ & $\begin{array}{c}\text { Evaluación } \\
\text { del servicio } \\
\text { recibido }\end{array}$ \\
\hline Tutorías & 98 & 16,7 & 8,0 \\
Actividades deportivas & 99 & 16,9 & 8,5 \\
Actividades artísticas & 70 & 11,9 & 8,8 \\
Idiomas & 63 & 10,7 & 8,5 \\
Atención médica & 39 & 6,60 & 7,9 \\
Atención psicológica & 35 & 5,96 & 8,6 \\
Apoyo académico a estudiantes indígenas & 6 & 1,02 & 8,7 \\
Cursos de educación continua & 32 & 5,45 & 8,0 \\
\hline
\end{tabular}




\section{Discusión y conclusiones}

De las cuatro dimensiones de autoeficacia evaluadas, solo en dos de ellas se encontraron diferencias significativas entre hombres y mujeres. Se observó que las mujeres reportaron mejores niveles de autoeficacia para resistir la presión ante el consumo de sustancias que los hombres. Posiblemente esto pueda explicarse porque socialmente se acepta más el consumo de sustancias en los hombres que en las mujeres, de ahí que ellas tengan una presión social más baja que la que se ejerce sobre los hombres. Este resultado destaca, tal y como lo proponen Dever \& Kim (2016), MacPhee et al., (2013) y Hen \& Goroshit (2014), la relevancia de considerar los factores de tipo cultural, social y psicológicos, los cuales, en este caso, dictan que tomar y fumar son conductas propias de los hombres. Este argumento es uno de los principales factores asociados a conductas de riesgo para los varones (Varela Arévalo et al., 2007; Pérez Padilla et al., 2010). Trabajar con la percepción de autoeficacia para aumentar la confianza y mejorar el control podría ser una opción para disminuir el riesgo de consumo de sustancias en la búsqueda de quedar bien o pasar momentos agradables con otros (Chicharro Romero et al., 2007; Feldman \& Kubota, 2015; Richardson et al., 2012).

Los resultados en relación con la participación en actividades deportivas y la autoeficacia deportiva de los estudiantes oscilan entre los niveles bajo y medio. Esto coincide con lo reportado por Varela et al. (2011) y permite identificar que el deporte no está presente como un indicador de un estilo de vida saludable en los participantes, lo que podría tener repercusiones a futuro en su calidad de vida (Hernández Zamora \& Romero Pedraza, 2010). Además, si las actividades depor- tivas y recreativas disminuyen los niveles de estrés y ansiedad, es posible que los universitarios que participaron en este estudio, al no estar realizándolas, tengan mayores dificultades para el manejo del estrés o que lo hagan a través de otros medios (Pulido Rull et al., 2011). Además, en la dimensión de autoeficacia deportiva los niveles reportados por las mujeres fueron inferiores a los de los hombres tal como sucedió en un estudio realizado por Abalde Amoedo \& Pino Juste (2016). Esta diferencia podría estar asociada a la creencia de que el deporte se relaciona con la masculinidad y no con la femineidad (Pavón Lores \& Moreno Murcia, 2008).

Entre las dimensiones en las que no se encontraron diferencias significativas, está la autoeficacia académica, cuyas similitudes coinciden con las reportadas por Carbonero Martín \& Merino Tejedor (2004). Sin embargo, estas contrastan con lo reportado en otras investigaciones, en las que sí han determinado diferencias significativas por sexo, cuyos niveles de autoeficacia académica en las mujeres resultaron significativamente más altos que en los hombres (Saunders et al., 2004).

En la dimensión de autoeficacia para enfrentar problemas se encontró que tanto hombres como mujeres reportaron puntajes altos. Estos resultados no concuerdan con los de Rosa-Rodríguez et al. (2015), quienes consignaron que las mujeres se evalúan con puntajes más altos que los hombres a nivel interpersonal; ni tampoco con lo reportado por González Barrón et al. (2002), quienes encontraron que las mujeres cuentan con más estrategias de afrontamiento que los hombres, pero que ellos tienen más habilidad para afrontar problemas. En tanto, Morales Rodríguez et al. (2013) reportaron que el tipo de afrontamiento que 
llegan a tener las mujeres es de tipo evasivo; también sugieren que las mujeres buscaban relacionarse positivamente con los demás con el propósito de obtener apoyo social y de sentir mayor bienestar, mientras que los hombres presentaban la tendencia de mantener relaciones positivas porque gozaban de un bienestar propio. Otra posible explicación es porque los hombres daban menor importancia a lo que los demás pensaban de ellos.

Con respecto a la participación social de los estudiantes en las actividades realizadas por la universidad, llama la atención que menos del $20 \%$ de los jóvenes reportó participar en los servicios de apoyo que se ofrecen a los estudiantes. Dado que recientemente se valora la calidad de la universidad por los servicios de apoyo que brinda, estos resultados no son favorables para la institución. En cuanto al uso de los servicios universitarios y el nivel de participación reportados en actividades recreativas, culturales y deportivas, se determinó que existe poco contacto y/o desconocimiento de la oferta de servicios que ofrece su universidad.

Con este trabajo es posible concluir, al igual que lo planteado por MacPhee et al. (2013), que el sistema de creencias de autoeficacia de mujeres y hombres varía en algunas dimensiones. Se puede observar que los hombres se consideran mejores y participan más en áreas que son consideradas culturalmente como propias masculinas, mientras que las mujeres presentan niveles bajos de autoeficacia y participan menos. Probablemente las creencias sociales sobre la eficacia de los hombres y las mujeres en determinadas áreas, repercuten sobre la percepción de autoeficacia de los estudiantes en diferentes campos del conocimiento y su correspondiente participación en los mismos.

Sin embargo, MacPhee et al. (2013) resaltan que las mujeres, con el paso del tiempo, mejoran su percepción de autoeficacia, pues aumentan su desempeño, lo que les permite obtener información sobre sí mismas y sus capacidades. Este contraste entre lo que ellas creen y lo que la realidad les retroalimenta contribuye a que paulatinamente vayan transformando su forma de autoevaluarse.

Como una limitación de este estudio y, a la vez, como una oportunidad de investigación a futuro se detecta la necesidad de realizar un análisis de las hipótesis propuestas a través de una estrategia basada en variables latentes como el modelamiento estructural para tener mayor capacidad explicativa de las variables involucradas, así como mayor precisión en la medición de las características psicométricas de los instrumentos utilizados.

Se espera que las instituciones de educación superior, a través de sus departamentos de atención estudiantil, estén promoviendo intervenciones dirigidas a disminuir la reprobación y la deserción de los estudiantes. Generar programas que contribuyan a incrementar la autoeficacia académica puede abonar a esa tarea. Por todo ello, se recomienda realizar una mayor difusión de los servicios universitarios, así como dar seguimiento a los mismos en cada una de las escuelas, facultades, centros e institutos, con el fin de promover una mayor participación social en las actividades académicas, culturales y sociales que ofrecen los diferentes programas universitarios y favorecer a su vez el incremento de las experiencias de autoeficacia. 


\section{Referencias}

Abalde Amoedo, N. \& Pino Juste, R. (2016). Evaluación de la autoeficacia y de la autoestima en el rendimiento deportivo en Judo. Retos. Nuevas Tendencias en Educación Física, Deporte y Recreación, 29, 109-113. Recuperado de http://www.redalyc.org/pdf/3457/345743464022.pdf

Abdul Gafoor, K. \& Muhammed Ashraf, P. (2012). Contextual influences on sources of academic self-efficacy: A validation with secondary school students of Kerala. Asia Pacific Education Review, 13(4), 607-616.

http://dx.doi.org/10.1007/s12564-012-9223-z

Aguirre, J. F., Blanco, J. R., Rodríguez-Villalobos, J. M., \& Ornelas, M. (2015). Autoeficacia general percibida en universitarios mexicanos, diferencias entre hombres y mujeres. Formación Universitaria, 8(5), 97-102. http://dx.doi.org/10.4067/S0718-50062015000500011

Alegre, A. A. (2013). Autoeficacia y procrastinación académica en estudiantes universitarios de Lima Metropolitana. Propósitos y Representaciones, 1(2), 57-82. http://dx.doi.org/10.20511/pyr2013.v1n2.29

Bandura, A. (1977). Self-efficacy: Toward unifying theory of behavior change. Psychological Review, 84(2), 191-215. http://dx.doi.org/10.1037/0033-295X.84.2.191

Bandura, A. (1986). Social foundations of thought and action: A social cognitive theory. Englewood Cliffs, New Jersey: Prentice Hall.

Blanco, H., Aguirre, J. F., Barrón, J. C., \& Blanco, J. R. (2016). Composición factorial de la Escala de Autoeficacia Académica en universitarios mexicanos. Formación Universitaria, 9(2), 81-88.

http://dx.doi.org/10.4067/S0718-50062016000200009

Blanco Vega, H., Ornelas Contreras, M., Aguirre Chávez J. F., \& Guedea Delgado, J. C. (2012). Autoeficacia percibida en conductas académicas: diferencias entre hombres y mujeres. Revista Mexicana de Investigación Educativa, 17(53), 557-571. Recuperado de https://is.gd/peURYU

Brenlla, M. E., Aranguren, M., Rossaro, M. F., \& Vázquez, N. (2010). Adaptación para Buenos Aires de la Escala de Autoeficacia General. Interdisciplinaria, 27(1), 77-94. Recuperado de http://www.scielo.org.ar/scielo.php?script=sci_arttext\&pid=S1668-70272010000100006

Caballero Domínguez, C. C., Hederich, C., \& Palacio Sañudo, J. E. (2010). El burnout académico: delimitación del síndrome y factores asociados con su aparición. Revista Latinoamericana de Psicología, 42(1), 131-146. Recuperado de http://www.redalyc.org/articulo.oa?id=80515880012

Cabanach, R. G., Valle, A., Rodríguez, S., Piñeiro, I., \& González, P. (2010). Las creencias motivacionales como factor protector del estrés en estudiantes universitarios. European Journal of Education and Psychology, 3(1), 75-87. Recuperado de http://www.redalyc.org/pdf/1293/129313736006.pdf 
Calvo Muñoz, I. \& Gómez Conesa, A. A. (2013). El fisioterapeuta y la educación para la salud en la escuela. Cuestiones de Fisioterapia: Revista Universitaria de Información e Investigación en Fisioterapia, 42(1), 1-2. Recuperado de

https://dialnet.unirioja.es/servlet/articulo?codigo $=4113060$

Carbonero Martín, M. A. \& Merino Tejedor, E. (2004). Autoeficacia y madurez vocacional. Psicothema, 16(2), 229-234. Recuperado de

http://www.psicothema.com/pdf/1187.pdf

Carrasco Ortiz, M. A. \& Del Barrio Gándara, M. V. (2002). Evaluación de la autoeficacia en niños y adolescentes. Psicothema, 14(2), 323-332. Recuperado de

http://www.psicothema.com/psicothema.asp?id=727

Chávez, A., Ornelas, M., De Rueda, B., \& Jasso, J. (2012). Invarianza factorial del Cuestionario sobre Creencias de Autoeficacia EACA en universitarios de ingeniería y ciencias sociales. Formación Universitaria, 5(3), 31-32.

http://dx.doi.org/10.4067/S0718-50062012000300005

Chicharro Romero, J., Pedrero Pérez, E. J., \& Pérez López, M. (2007). Autoeficacia para resistirse al consumo de sustancias como predictora de resultados de tratamiento y su relación con variables de personalidad: estudio de una muestra de adictos con el DTCQ, el VIP y el MCMI-II. Adicciones, 19(2), 141-151.

http://dx.doi.org/10.20882/adicciones.312

Dapelo Pellarano, B. \& Matus Jara, M. (2013). Necesidades de fortalecimiento cognitivomotivacional en estudiantes universitarios de primer año: implicaciones para una nivelación efectiva de competencias. Revista de Orientación Educacional, 27(52), 15-33. Recuperado de http://www.roe.cl/index.php/roe/article/view/29/23

Dever, B. V. \& Kim, S. Y. (2016). Measurement equivalence of the PALS Academic SelfEfficacy Scale. European Journal of Psychological Assessment, 32(1), 61-67. http://dx.doi.org/10.1027/1015-5759/a000331

Espada, J. P, González, M. T., Orgilés, M., Carballo, J. L., \& Piqueras, J. A. (2012). Validación de la Escala de Autoeficacia General con adolescentes españoles. Electronic Journal of Research in Educational Psychology, 10(26), 355-370. Recuperado de http://www.redalyc.org/articulo.oa?id=293123551018

Estrada Ruiz, M. J. (2010). Jóvenes y participación social en la educación. Una experiencia en el nivel medio superior de Chiapas, México. REICE. Revista Iberoamericana sobre Calidad, Eficacia y Cambio en Educación, 8(3) 149-166. Recuperado de http://www.redalyc.org/articulo.oa?id=55115052010

Feldman, D. B. \& Kubota, M. (2015). Hope, self-efficacy, optimism, and academic achievement: Distinguishing constructs and levels of specificity in predicting college grade-point average. Learning and Individual Differences, 37, 210-216. http://dx.doi.org/10.1016/j.lindif.2014.11.022

Flores León, A., González-Celis Rangel, A. L., \& Valencia Ortiz, A. (2010). Validación del Instrumento de Autoeficacia para Realizar Conductas Saludables en niños mexicanos sanos. Psicología y Salud, 20(1), 23-30. Recuperado de http://www.uv.mx/psicysalud/psicysalud-20-1/20-1/araceli-flores-leon.pdf 
González Barrón, R., Montoya Castilla, I., Casullo M. M., \& Bernabéu Verdú, J. (2002). Relación entre estilos y estrategias de afrontamiento y bienestar psicológico en adolescentes. Psicothema, 14(2), 363-368. Recuperado de http://www.psicothema.com/psicothema.asp?id=733

González-Betanzos, F., Rivera-Heredia, M. E. \& García-Rangel, F. (2016). Indicadores de riesgo de deserción en estudiantes de licenciatura. En M. L. VargasGarduño, M. I. Gómez del Campo del Paso, \& A. D. Vargas-Silva (Eds.), Escenarios contemporáneos de la psicología. Controversias y desafíos (pp. 311-322). Morelia, México: Universidad Michoacana de San Nicolás de Hidalgo.

Hackett, G. \& Betz, N. E. (1981). A self-efficacy approach to the career development of women. Journal of Vocational Behavior, 18(3), 326-339. http://dx.doi.org/10.1016/0001-8791(81)90019-1

Hen, M. \& Goroshit, M. (2014). Academic procrastination, emotional intelligence, academic self-efficacy, and GPA: A comparison between students with and without learning disabilities. Journal of Learning Disabilities, 47(2), 116-124. http://dx.doi.org/10.1177/0022219412439325

Hernández Zamora, Z. E. \& Romero Pedraza, E. (2010). Estrés en personas mayores y estudiantes universitarios: un estudio comparativo. Psicología Iberoamericana, 18(1), 56-68. Recuperado de http://www.redalyc.org/articulo.oa?id=133915936007

Komarraju, M. \& Nadler, D. (2013). Self-efficacy and academic achievement: Why do implicit beliefs, goals, and effort regulation matter? Learning and Individual Differences, 25, 67-72.

http://dx.doi.org/10.1016/j.lindif.2013.01.005

Lira-Mandujano, J., González-Betanzos, F., Carrascoza Venegas, C. A., Ayala V., H. E., \& Cruz-Morales, S. E. (2009). Evaluación de un programa de intervención breve motivacional para fumadores: resultados de un estudio piloto. Salud Mental, 32(1), 35-41. Recuperado de https://is.gd/WaybOF

López González, R. \& Anaya Rodríguez, R. (2016). Estudiantes universitarios interactuando en red: ¿nuevos escenarios de interacción, expresión y participación ciudadana? Revista Interamericana de Educación de Adultos, 38(1), 48-67. Recuperado de http://www.redalyc.org/articulo.oa?id=457545337004

MacPhee, D., Farro, S., \& Canetto, S. S. (2013). Academic self-efficacy and performance of underrepresented STEM majors: Gender, ethnic, and social class patterns. Analyses of Social Issues and Public Policy, 13(1), 347-369. http://dx.doi.org/10.1111/asap.12033

Morales Rodríguez, M., Benítez Hernández, M., \& Agustín Santos, D. (2013). Habilidades para la vida (cognitivas y sociales) en adolescentes de una zona rural. Revista Electrónica de Investigación Educativa, 15(3), 98-113. Recuperado de http://redie.uabc.mx/vol15no3/contenido-moralesetal.html 
Morony, S., Kleitman, S., Ping Lee, Y., \& Stankov, L. (2013). Predicting achievement: Confidence vs self-efficacy, anxiety, and self-concept in Confucian and European countries. International Journal of Educational Research, 58, 79-96. http://dx.doi.org/10.1016/j.ijer.2012.11.002

Pavón Lores, A. \& Moreno Murcia, J. A. (2008). Actitud de los universitarios ante la práctica físico-deportiva: diferencias por géneros. Revista Psicología del Deporte, 17(1), 723. Recuperado de http://www.rpd-online.com/article/viewFile/245/245

Peinado, J. E., Ornelas, M., Blanco, J. R., \& González, M. A. (2015). Invarianza factorial de la Escala de Autoeficacia en la Solución de Problemas y Comunicación en estudiantes universitarios. Formación Universitaria, 8(4), 85-91.

http://dx.doi.org/10.4067/S0718-50062015000400010

Perales Mejía, F. J. \& Escobedo Carrillo, M. M. (2016). La participación social en la educación: entre propuestas innovadoras y disposiciones históricas. Revista Electrónica de Investigación Educativa, 18(1), 69-81. Recuperado de

http://redie.uabc.mx/redie/article/view/738

Pérez, E. R. \& Cupani, M. (2008). Validación del Inventario de Autoeficacia para Inteligencias Múltiples Revisado (IAMI-R). Revista Latinoamericana de Psicología, 40(1), 47-58. Recuperado de http://www.redalyc.org/pdf/805/80500104.pdf

Pérez Moreno, Y. \& Álvarez Hernández, J. (2015). Factores motivacionales que influyen en el desarrollo de los juegos deportivos interfacultades de la Universidad de Antioquia. Revista de Educación Física, 4(4), 45-56. Recuperado de https://is.gd/FCz3NM

Pérez Padilla, M. L., Ponce Rojo, A., Hernández Contreras, J., \& Márquez Muñoz, B. A. (2010). Salud mental y bienestar psicológico en los estudiantes universitarios de primer ingreso de la Región Altos Norte de Jalisco. Revista de Educación y Desarrollo, 14(1), 31-37. Recuperado de http://www.cucs.udg.mx/revistas/edu_desarrollo/anteriores/14/014_Perez.pdf

Pulido Rull, M. A., Serrano Sánchez, M. L., Valdés Cano, E., Chávez Méndez, M. T., Hidalgo Montiel, P., \& Vera García, F. (2011). Estrés académico en estudiantes universitarios. Psicología y Salud, 21(1), 33-37. Recuperado de https://is.gd/iAjIj1

Qualter, P., Dacre Pool, L., Gardner, K. J., Ashley-Kot, S., Wise, A., \& Wols, A. (2015). The Emotional Self-Efficacy Scale: Adaptation and validation for young adolescents. Journal of Psychoeducational Assessment, 33(1), 33-45. http://dx.doi.org/10.1177/0734282914550383

Reigal Garrido, R. E., Videra García, A., Martín Tamayo, I., \& Juárez Ruiz de Mier, R. (2013). Importancia del autoconcepto físico y la autoeficacia general en la predicción de la conducta de práctica física. Apunts. Educación Física y Deportes, 112, 46-51. http://dx.doi.org/10.5672/apunts.2014-0983.es.(2013/2).112.03 
Rhodes, J. E. \& Jason, L. A. (1990). A social stress model of substance abuse. Journal of Consulting and Clinical Psychology, 58(4), 395-401. Recuperado de http://www.ncbi.nlm.nih.gov/pubmed/2212175

Richardson, M., Abraham, C., \& Bond, R. (2012). Psychological correlates of university students' academic performance: A systematic review and meta-analysis. Psychological Bulletin, 138(2), 353-387. http://dx.doi.org/10.1037/a0026838

Rivera Heredia, M. E., Obregón Velasco, N., \& Ayala Mira, M. (2012). Diagnóstico de la condición y posición de género en el Municipio de Marcos Castellanos, Michoacán. Morelia, México: Facultad de Psicología de la Universidad Michoacana de San Nicolás de Hidalgo. Recuperado de

https://is.gd/cWJIte

Rivera Heredia, M. E. \& Pérez Padilla, M. L. (2012). Evaluación de los recursos psicológicos. Uaricha, 9(19), 1-19. Recuperado de

https://is.gd/ykAjE8

Rosa-Rodríguez, Y., Negrón Cartagena, N., Maldonado Peña, Y., Quiñones Berrios, A., \& Toledo Osorio, N. (2015). Dimensiones de bienestar psicológico y apoyo social percibido con relación al sexo y nivel de estudio en universitarios. Avances en Psicología Latinoamericana, 33(1), 31-43.

http://dx.doi.org/10.12804/apl33.01.2015.03

Rouxel, G. (1999). Path analyses of the relations between self-efficacy, anxiety and academic performance. European Journal of Psychology of Education, 14(3), 403-421. Recuperado de http://www.jstor.org/stable/23420332

Saunders, J., Davis, L., Williams, T., \& Williams, J. H. (2004). Gender differences in selfperceptions and academic outcomes: A study of African-American high school students. Journal of Youth and Adolescence, 33(1), 81-90. http://dx.doi.org/10.1023/A:1027390531768

Stankov, L., Lee, J., Luo, W., \& Hogan, D. J. (2012). Confidence: A better predictor of academic achievement than self-efficacy, self-concept and anxiety? Learning and Individual Differences, 22(6), 747-758. http://dx.doi.org/10.1016/j.lindif.2012.05.013

Varela, M. T., Duarte, C., Salazar, I. C., Lema, L. F., \& Tamayo, J. A. (2011). Actividad física y sedentarismo en jóvenes universitarios de Colombia: prácticas, motivos y recursos para realizarlas. Colombia Médica, 42(3), 269-277. Recuperado de http://www.bioline.org.br/request?rc11049

Varela Arévalo, M. T., Salazar Torres, I. C., Cáceres de Rodríguez, D. E., \& Tovar Cuevas, J. R. (2007). Consumo de sustancias psicoactivas ilegales en jóvenes: factores psicosociales asociados. Pensamiento Psicológico, 3(8), 31-45. Recuperado de http://www.redalyc.org/pdf/801/80130804.pdf 
Weng, F., Cheong, F., \& Cheong, C. (2010). The combined effect of self-efficacy and academic integration on higher education students studying IT majors in Taiwan. Education and Information Technologies, 15(4), 333-353. http:/dx.doi.org/10.1007/s10639-009-9115-y

Fecha de recepción: 20 de septiembre de 2016 Fecha de aceptación: 29 de noviembre de 2016 